\title{
Trompetenbäume (Catalpa): Tropisch anmutende Blütensolitäre
}

\author{
Veit Martin Dörken \& Annette Höggemeier
}

\begin{abstract}
Catalpas are ornamental trees with spectacular flowers, large leaves and interesting fruits, which persist over the winter. They are solitary trees for huge gardens and parks.
\end{abstract}

\section{Zusammenfassung}

Trompetenbäume (Catalpa) sind exotische Bäume, die mit ihrem Blütenflor, ihren riesigen, tropisch anmutenden Blättern und lange am Individuum verbleibenden Früchten interessante Solitärgehölze für große Gärten und Parkanlagen darstellen.

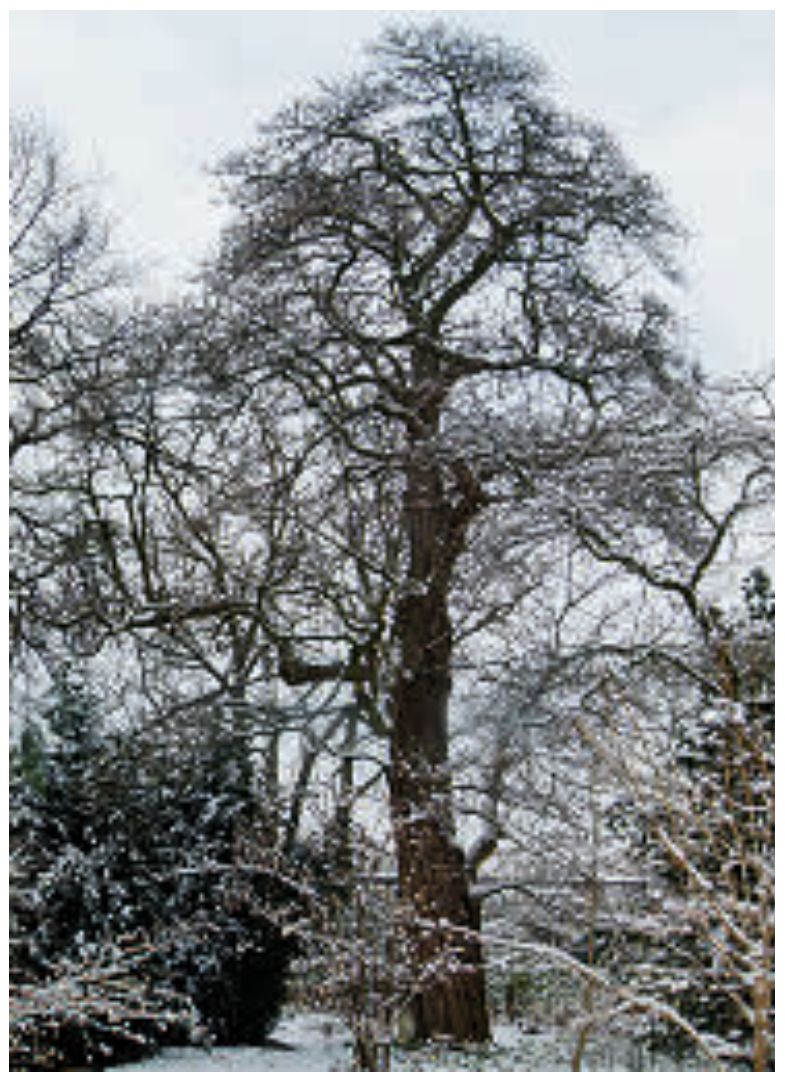

\section{Einleitung}

Trompetenbäume (Catalpa-Arten, Bignoniaceae) sind wertvolle Park- und Hausbäume. Sie sind nicht nur wegen ihres massenhaften Blütenflors attraktiv, sondern auch wegen ihrer großen, exotisch anmutenden Blätter sowie des ausdauernden Fruchtbehangs wertvolle und attraktive Solitärgehölze.

Die botanische Bezeichnung Catalpa ist die englische Form der regionalen Bezeichnung „kuthulpa“ (SAuerhoff 2003). Die sehr disjunkt verbreitete Gattung der Trompetenbäu- me umfasst rund zehn Arten, die in Ostasien, auf den Westindischen Inseln und im südöstlichen Nordamerika heimisch sind. In Mitteleuropa gedeiht insbesondere $C$. bignonioides sehr gut. In klimatisch begünstigten Bereichen sind bereits erste Verwilderungen bekannt.

Die Gattung Catalpa ist neben der kletternden Gattung Campsis (Trompetenblume) eine der wenigen Vertreter der Bignoniaceae, die auch bei uns in Mitteleuropa an geschützteren Standorten ausreichend frosthart sind. Besonders Catalpa bignonioides findet man häufiger in Parkanlagen und wird gelegentlich auch als Hausbaum gepflanzt.

\section{Kulturansprüche}

Trompetenbäume sind etwas frostempfindlich und benötigen einen geschützten Standort. In besonders kalten Wintern kann es dazu kommen, dass sie bis ins alte Holz zurückfrieren. Besonders in der Jugend reagieren Trompetenbäume empfindlich auf zu tiefe winterliche Temperaturen. Beachtet man jedoch die generellen Standortansprüche wie einen durchlässigen, sandig-lehmigen Boden in sonnig-warmer Lage, gewährleistet dies das ausreichende und vor allem auch rechtzeitige Ausreifen der Triebe. Eine Bodenbedeckung verhilft besonders jungen Individuen zu einer erhöhten Frosttoleranz (EISELt \& SCHRÖDER 1977). Da es sich bei den Trompetenbäumen um Arten aus Auenregionen und Niederungen handelt, sollte dies auch in deren Kultur berücksichtigt werden. So müssen die Substrate ausreichend feucht, jedoch gut drainiert und nährstoffreich sein 
(B̈̈RTELS 2001). Generell sollten Trompetenbäume, wie auch alle anderen großlaubigen $\mathrm{Ar}$ ten, nicht an zu exponierten Standorten gepflanzt werden, da die großflächigen, weichen Blätter durch starken Wind leicht zerrissen werden können (Kelly \& Hillier 2004). Auf zunehmenden Schattendruck reagieren Trompetenbäume mit einem ausgeprägten Schiefwuchs (B̈̈rTELS 2001).

\section{Morphologie}

Die steiftriebigen, winterkahlen Trompetenbäume sind Großsträucher bis rundkronige Kleinbäume, nur wenige Arten wachsen großbaumartig (EISELt \& Schröder 1977). Besonders junge Individuen können jährlich enorme Zuwächse erlangen. Im Alter entwickeln die kurzstämmigen Bäume eine dichte, weit ausladende Krone. Ihre Borke ist dunkelbraun und löst sich meist in kleinen Platten vom Stamm. Die Winterknospen sind im Verhältnis zur Blattnarbe klein und unauffällig. Die gegenständig angeordneten, großen Blätter sind artspezifisch ganzrandig oder leicht gelappt. Alle Trompetenbäume treiben sehr spät aus, teilweise erst im Juni. Die Herbstfärbung ist meist unauffällig fahlgelb und ohne nennenswerten Zierwert. Häufiger erfolgt bereits ein Abwurf der noch grünen, ungefärbten Blätter, wenn diese den ersten Herbstfrösten ausgesetzt wurden. Dies ist ein Hinweis auf die schwerpunktmäßig subtropische bis tropische Verbreitung der Bignoniaceae, zu denen Catalpa gehört. Die aufrechten, bis $30 \mathrm{~cm}$ langen Rispen stehen endständig. Die fünf kleinen Kelchblätter sind röhrig miteinander verwachsen und die fünf Kronblätter bilden eine schiefe Glocke. Die Kronröhre ist fünflappig. Dabei stehen die zwei kleineren Lappen oben (median hinten), die drei größeren unten (median vorne). Der Kronblattsaum ist artspezifisch deutlich gewellt

Abb. 1 (Seite 26): Habitus eines alten Exemplares von Catalpa speciosa im Palmengarten.

Abb. 2 (oben): Habitus von Catalpa ovata.

Abb. 3 (unten): Blütenstand von Catalpa ovata.

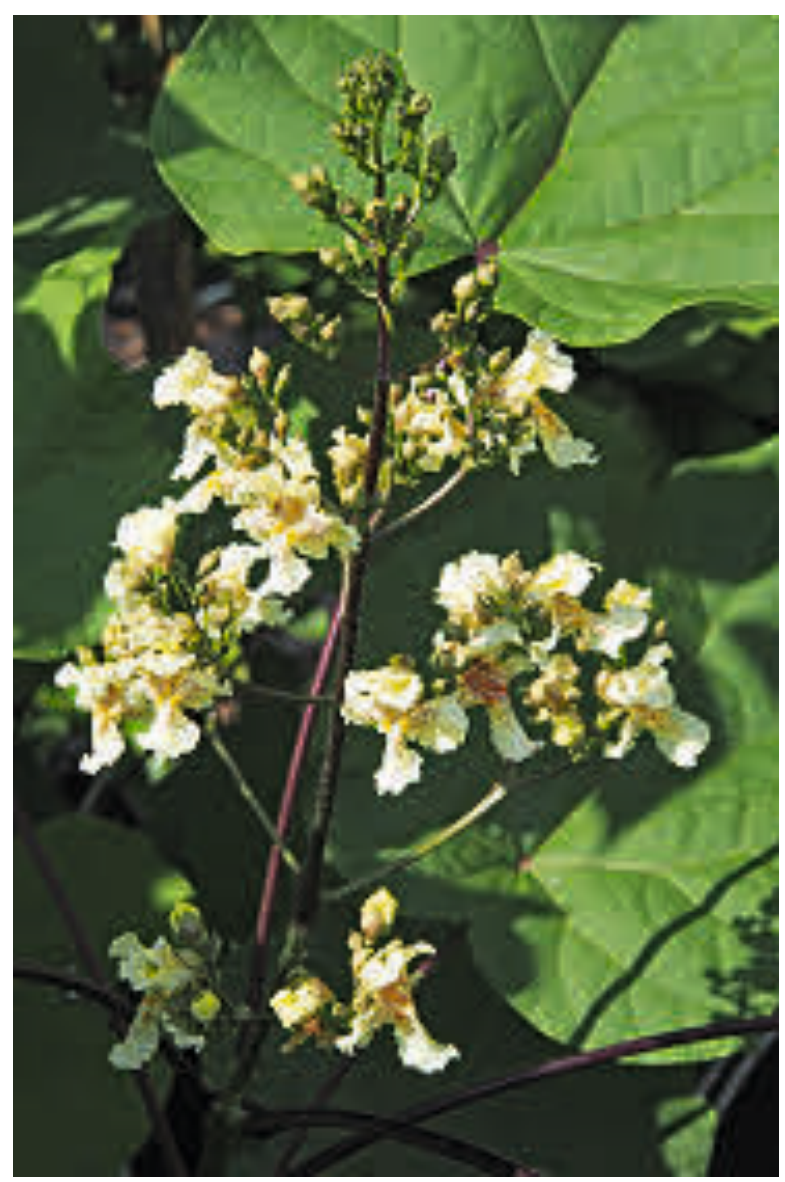




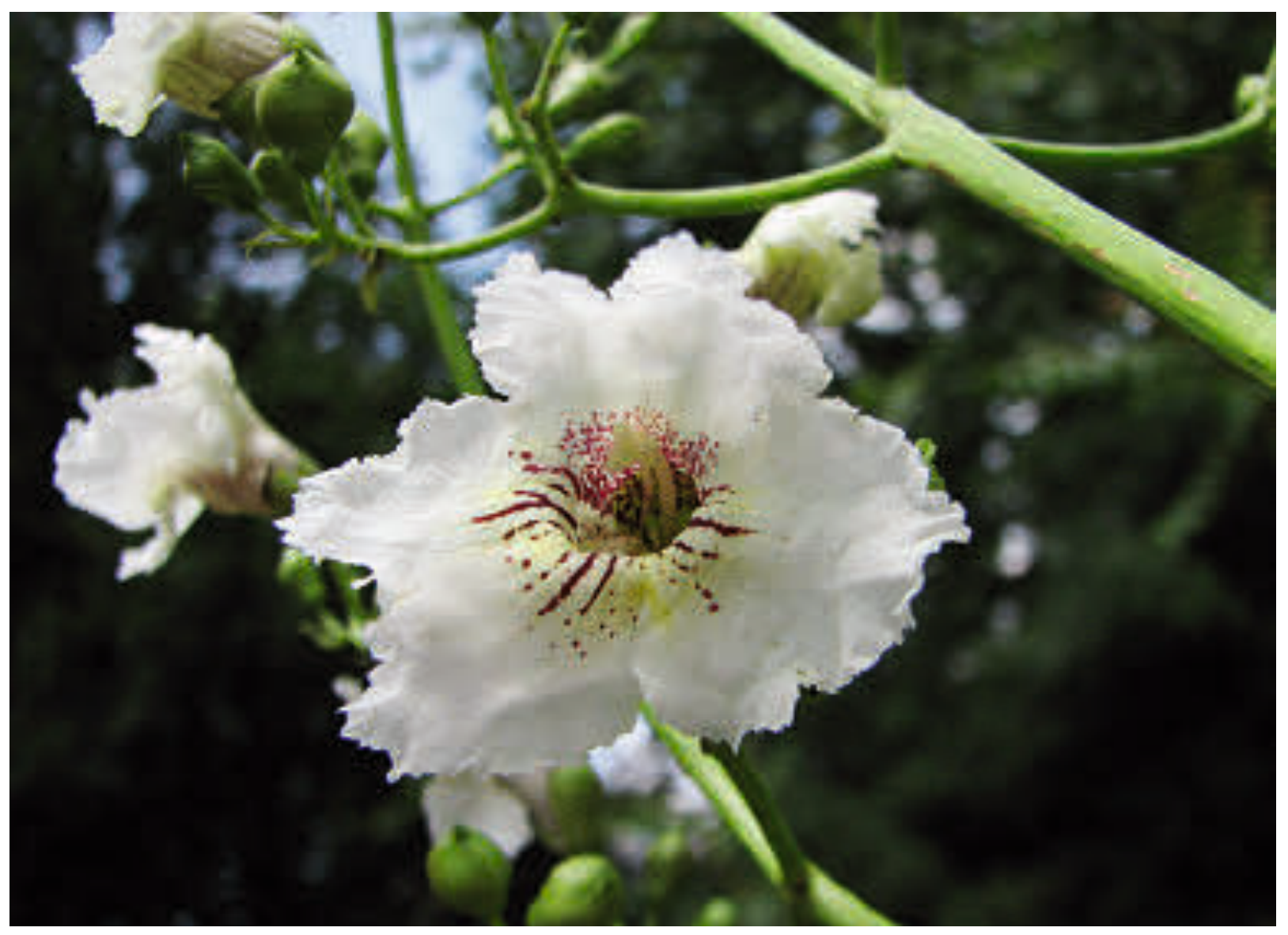

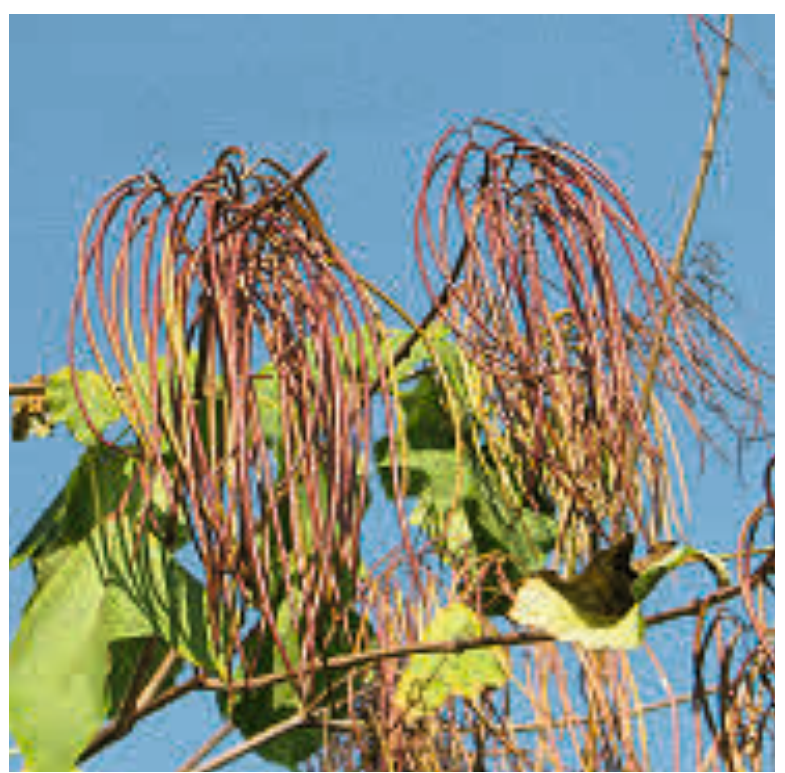

bis gefranst. Die untereinander freien Staubblätter sind auf zwei reduziert und mit den Kronblättern verbunden. Der oberständige Fruchtknoten baut sich aus zwei Fruchtblättern auf. Die Früchte, bis $40 \mathrm{~cm}$ lange, dunkelbraune, schmal längliche Kapseln, bleiben z.T. bis zum nächsten Frühjahr am Baum hängen. Sie enthalten zahlreiche, bis $5 \mathrm{~cm}$ lange, geflügelte Samen, die vom Wind ausgebreitet werden.

Trompetenbäume sind als schwach giftig einzustufen. Sie enthalten Catalpin, das mit Ausnahme der Samen in der gesamten Pflanze vorkommt.

\section{Häufig gepflanzte Arten 4.1 Catalpa bignonioides WALTER - Gewöhnlicher Trompetenbaum}

Diese Art ist in den südöstlichen USA (Georgia und Florida bis Lousiana) heimisch. In einigen weiteren südöstlichen Staaten wie in Texas gilt sie als eingebürgert. Der Gewöhnliche Trompetenbaum wird 10 bis $15 \mathrm{~m}$ hoch. Auffällig ist der verhältnismäßig dicke, kurze Stamm. Die bis $30 \mathrm{~cm}$ langen, kurz zugespitzten Blätter sind breiteiförmig und ganzrandig. Die Blattbasis ist schwach herzförmig bis abgerundet, selten gelappt. Die Blattunterseite ist heller und meist dicht behaart. Zerrieben verströmen die Blätter einen unangenehmen, intensiven Geruch. Der Blattstiel ist kahl und leicht abgeflacht. Die rein 


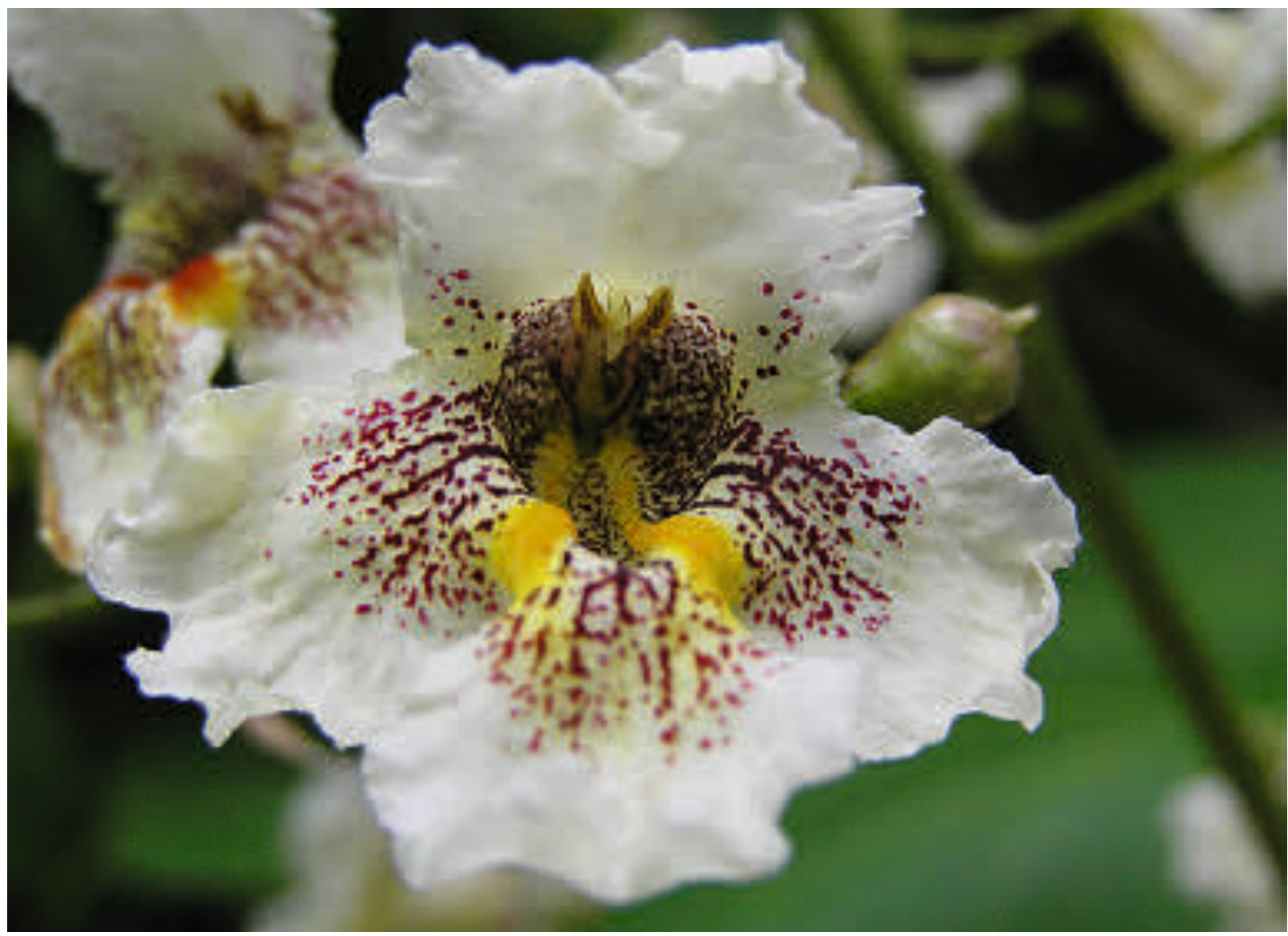

weißen, bis $5 \mathrm{~cm}$ langen Blüten erscheinen von Juni bis Juli in lockeren, bis $15 \mathrm{~cm}$ langen Rispen und haben einen sehr schiefen und gekräuselten bis gefransten Saum. Der Schlundbereich ist durch zahlreiche kleine gelbe und purpurfarbene Flecken sowie zwei gelbe Längsstreifen gezeichnet. In seiner Heimat wird C. bignonioides u.a. wegen seines hohen Nutzholzwertes (Herstellung von Zaunpfählen) geschätzt (Heywood 1978). Vom Gewöhnlichen Trompetenbaum werden besonders häufig die Sorten 'Aurea' (Großstrauch bis Kleinbaum mit leuchtend gold-gelb gefärbtem Laub), 'Nana' (dicht verzweigte, kleinblätterige Kugelform, Krone im Alter flachrund, in der Regel als Hochstamm veredelt und stets blütenlos) und 'Variegata' (Großstrauch bis Kleinbaum mit cremeweißer Blattpanaschierung) gepflanzt.

\subsection{Catalpa ovata G. Don - Gelber Trompetenbaum}

Diese aus Westchina stammende Art wird $15 \mathrm{~m}$ hoch. Die Blätter sind breiteiförmig mit mehr

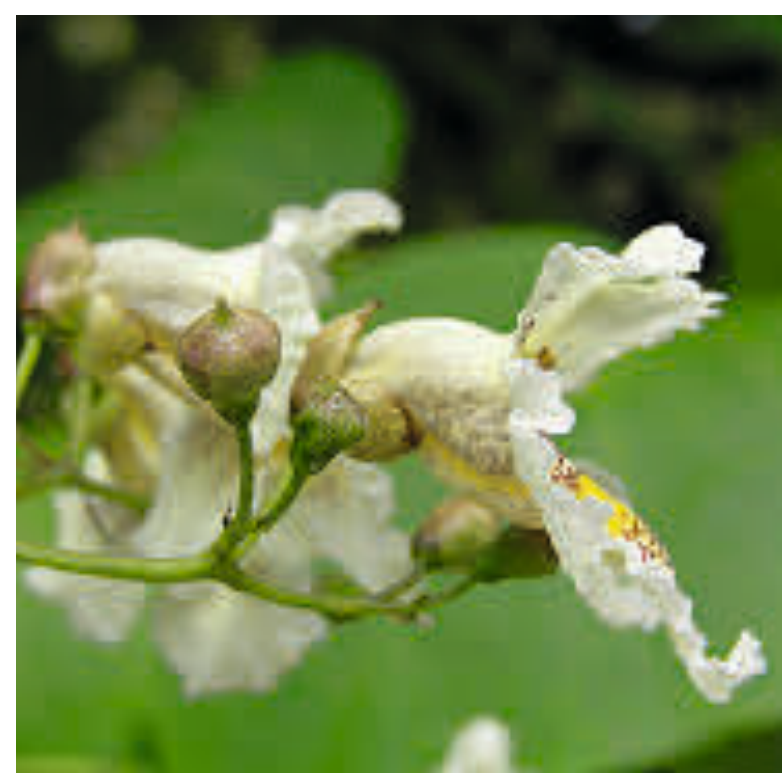

Abb. 4 (Seite 28 oben): Blüte von Catalpa ovata.

Abb. 5 (Seite 28 unten): Fruchtender Zweig von Catalpa ovata.

Abb. 6 (oben): Frontalansicht einer Blüte von Catalpa speciosa.

Abb. 7 (unten): Seitenansicht einer Blüte von Catalpa speciosa. 


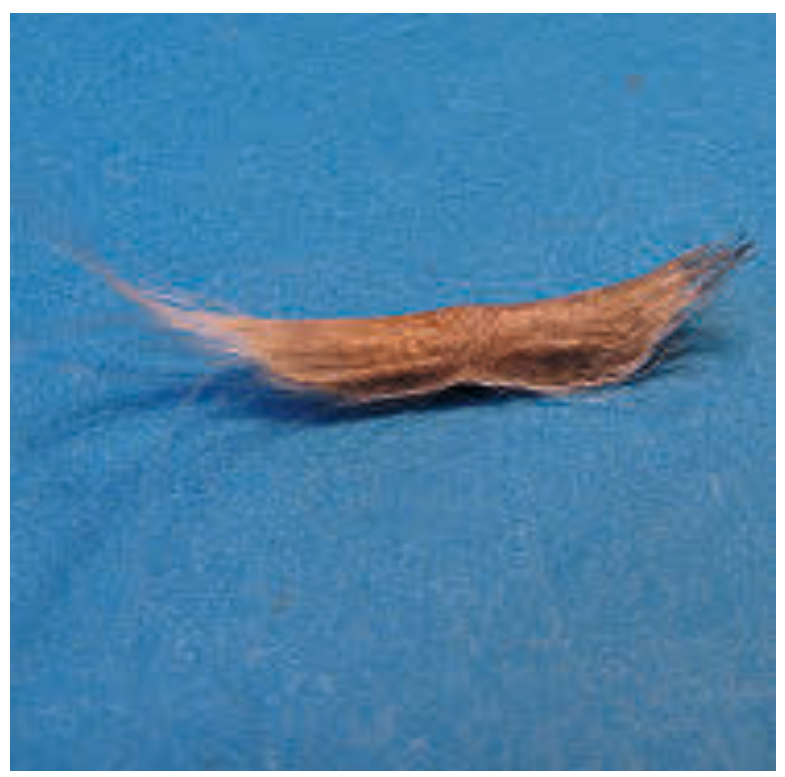

oder weniger stark herzförmiger Basis und meist deutlich drei- bis fünflappig. Die Lappen sind zugespitzt. Die Blattunterseite oder zumindest die Nerven sind behaart. Junge Blätter sind im Austrieb dunkel purpurrot gefärbt, später jedoch auffällig dunkel olivgrün. Der rötliche Blattstiel ist steifbehaart. Zerrieben, verströmen die Blätter wie auch die von C. bignonioides einen sehr intensiven, unangenehmen Geruch. Die kleinen 1,5-2,5 cm langen, cremeweißen bis hellgelblichen Blüten erscheinen im Juli und stehen in bis zu $20 \mathrm{~cm}$ langen Rispen. Die Kronröhre weist auf der Innenseite zahlreiche violette Flecken sowie zwei gelbliche und später rötliche Streifen auf. Der Kronsaum ist gekräuselt. Die dickwandigen Kapseln werden bis $30 \mathrm{~cm}$ lang.

\subsection{Catalpa speciosa (WARder ex BARNEY) ENGLM. - Prächtiger Trompetenbaum}

Ein weiterer, häufig gepflanzter Vertreter ist $\mathrm{Ca}$ talpa speciosa aus den USA. Es handelt sich um den attraktivsten der bei uns gepflanzten Trompetenbäume. Er ist besonders frosthart (KRÜssmann 1977). Der Prächtige Trompetenbaum wird bis $30 \mathrm{~m}$ hoch und hat im Alter eine im Vergleich zu den beiden zuvor beschriebenen Arten eine relativ schlanke Krone. Die Borke ist deutlich tief gefurcht. Die bis $30 \mathrm{~cm}$ langen Blätter sind breiteiförmig bis länglich mit deutlich ausgezogener Spitze. Die Basis ist meist sehr tief herzförmig, gelegentlich gestutzt. Der Blattrand ist bei fast allen Blättern ganzrandig, nur gelegentlich werden zwei Basallappen ausgebildet. Die Blattunterseite ist dicht behaart. Die zerriebenen Blätter sind ohne auffälligen Geruch. Die weißen Blüten duften und sind darüber hinaus ein unübersehbarer Blickfang, obwohl sie auch bei dieser Art erst nach dem Laubaustrieb Ende Juni bis Anfang Juli erscheinen. Sie stehen in bis zu $20 \mathrm{~cm}$ langen, aufrechten, sehr locker verzweigten und im Vergleich zu C. bignonioides wenigblütigen Rispen. Die Einzelblüte wird $5-6 \mathrm{~cm}$ breit und hat nur einen leicht schiefen Saum. Im Blüteninneren sind zwei deutliche gelbe Längsstreifen sowie zahlreiche, jedoch kleine, dunkel purpurfarbene Flecken zu erkennen. Die dickwandigen Früchte werden bis $40 \mathrm{~cm}$ lang. Gelegentlich wird im Handel die weiß oder gelb panaschierte Sorte 'Albovariegata' angeboten.

Attraktive Trompetenbäume sind auch im Palmengarten zu finden, so z. B. ein stattliches und altes Exemplar von $C$. speciosa gegenüber des Blütenhauses und $C$. bignonioides, passend zum Namen des Baumes, im Konzertgarten.

\section{Literatur}

Bärtels, A. 2001: Enzyklopädie der Gartengehölze. Stuttgart.

Dörken, V. M. \& Höggemeier, A. 2009: Botanischdendrologische Streifzüge. Gehölzführer des Botanischen Gartens Bochum. - Bochum.

Eiselt, M. G. \& Schröder, R. 1977: Die Laubgehölze. - Leipzig.

Heywood, V. H. 1982: Blütenpflanzen der Welt. - Basel. Kelly, J. \& Hillier, J. 2004: Bäume und Sträucher, 2. Aufl. - Braunschweig.

Krüssmann, G. 1976: Handbuch der Laubgehölze, Band 1: A-D. - Berlin, Hamburg.

SAuerhoff, F. 2003: Etymologisches Wörterbuch der Pflanzenamen. Die Herkunft der wissenschaftlichen, deutschen, englischen und französischen Pflanzennamen. - Stuttgart.

Abb. 8 (oben): Die Samen von Catalpa-Arten werden durch den Wind ausgebreitet. Sie sind sehr leicht und an den Rändern behaart. Hier ein Same von Catalpa bignonioides. 